\title{
Harmonic Analysis of Power System Signals using A New Regularized Adaptive Windowed Lomb Periodogram
}

\author{
Z. G. Zhang and S. C. Chan \\ Department of Electrical and Electronic Engineering \\ The University of Hong Kong, Pokfulam Road, Hong Kong, China \\ \{zgzhang; scchan\}@eee.hku.hk
}

\begin{abstract}
This paper proposes a new regularized adaptive windowed Lomb periodogram (RAWLP) method for timefrequency analysis of non-stationary power signals. It extends the conventional Lomb periodogram by estimating the periodogram locally using the weighted least-squares (WLS) estimator. Instead of employing one constant window in WLS, variable window bandwidth is adaptively selected by the intersection of confidence intervals (ICI) method to achieve a better tradeoff between time resolution and frequency resolution. Furthermore, regularization techniques are incorporated in the AWLP to further improve its performance by reducing the variance of the estimator. Simulation results show that the proposed RAWLP method has superior performance over windowed Lomb periodogram with one constant bandwidth for estimating the harmonic and interharmonic frequencies in power systems.
\end{abstract}

\section{INTRODUCTION}

Time-frequency analysis (TFA) techniques aim to discover time-variant spectral features of nonstationary signals, and they have been extensively studied and successfully applied in a wide variety of fields, such as biomedical engineering and speech processing [1]-[3]. Recently, there is an increasing interest in TFA of voltage and current signals in power systems and related energy areas [4]-[7]. TFA techniques can effectively reveal useful information and status of power systems for stable operation. For example, by estimating the time-variant frequency and magnitude of harmonic and interharmonic components, the quality can be effectively analyzed. Therefore, TFA techniques have been proposed recently to address a variety of problems in power quality assessment and power system diagnostics [7].

Although a number of TFA methods have been proposed for analyzing power measurements with different performances, the fundamental time/frequency resolution tradeoff problem has not been satisfactorily addressed. This problem is concerned with the selection of a proper window or kernel size to provide the best tradeoff between time resolution and frequency resolution in analyzing nonstationary signals. For instance, in short-time Fourier transform (STFT), the window size should be appropriately chosen to compromise between time resolution and frequency resolution. The wavelet transform (WT) addresses the window selection problem by applying a short window at high frequencies and a long window at low frequencies. However, WT suffers from degraded frequency resolution for highfrequency components and a degraded time resolution for lowfrequency components. Since the power signals typically consist of a number harmonic/interharmonic components with different time-frequency characteristics, it is desirable that the window be adaptive in time-frequency domain in accordance with the local time-frequency characteristics of the signals to be analyzed. For example, a long time window is desirable for analyzing slowly-varying harmonics, while a small window is preferred for tracking fast-varying harmonics.

In this paper, we propose a regularized adaptive windowed Lomb periodogram (RAWLP) method to address the time/frequency resolution tradeoff problem in the harmonic analysis of power system signals. The RAWLP method is an extension of the Lomb periodogram, which was first proposed for astronomical data [8], [9]. Lomb periodogram has also been successfully adopted for analyzing other types of signals, such as heart rate variability [10] and genomic data [11]. The basic idea of Lomb periodogram is to estimate the amplitude of a given sinusoid with a certain frequency based on a least-squares (LS) fitting of the sinusoid to the data samples. Since the conventional Lomb periodogram is obtained by fitting the whole data block to the sinusoidal model, it cannot reveal the time-varying spectrum of nonstationary signals. To address the problem, we proposed a windowed version of the Lomb periodogram, called the windowed Lomb periodogram (WLP) in [12]. The WLP method employs a sliding window with appropriate sizes to split the signal into short-time segments and estimating the Lomb periodogram of each data segment, which can be estimated by a weighted least-squares (WLS) fitting of the windowed data to the sinusoid. By recognizing the fact that the WLP estimator is identical to the identification of a time-varying linear regression model with the amplitudes of the given sinusoid as the model coefficients, the asymptotic bias and variance of WLP for a time-varying sinusoid signal can be established. Moreover, it suggests that there is an optimal window size or bandwidth which can minimize the mean squared error (MSE).

Similar to the STFT, the selection of the window length poses an important problem for WLP. For slowly-varying harmonics, a long time window is suitable because more samples can be used to determine their amplitudes and hence better accuracy is obtained. The reduced variances of the spectral coefficients give a better frequency resolution at the expense of a lower time resolution. On the contrary, for fast varying harmonic components, a small window can effectively reduce the estimation bias introduced by remote and uncorrelated data. As a result, a better time resolution is

This study was partially supported by the University of Hong Kong CRCG Small Project Funding. 
achieved for tracking these fast-varying components at the cost of a lower frequency resolution. The time-frequency resolution tradeoff problem of WLP originates from the biasvariance tradeoff problem of the WLS fitting problem. Though there exists an optimal local bandwidth which minimizes the MSE, it is difficult to be used in practice since some of the quantities are unknown and difficult to be estimated. Therefore, we next propose to adopt an intersection of confidence intervals (ICI)-based bandwidth selection method [12]-[16], which is a popular adaptive bandwidth selection method, for adaptively choosing the window size of our WLP. This yields a new adaptive windowed Lomb periodogram (AWLP).

Furthermore, to lower the estimation variance of the AWLP, we propose a new regularized AWLP (RAWLP) method by imposing a regularization term in the WLS objective function. Regularization techniques have received increasing interest recently in a wide variety of fields, because of its effectiveness in reducing the estimation variance for automatic model selection. Some regularization techniques, such as the $L_{1}$ regularization and the smoothly clipped absolute deviation (SCAD) regularization, can also possess the sparsity property where small and irrelevant coefficients can be shrunk towards zero [17]. This is desirable for analyzing power system, where few sinusoids are presence, using the AWLP. With regularization, the WLSbased estimator will exhibit a substantially lower variance, and so will the resultant time-frequency representation (TFR). Several popular regularization techniques, such as $L_{2}$ regularization [16], $L_{1}$ regularization [19], and SCAD [17], are evaluated and compared in the paper. The SCAD regularization is recommended since it is an asymptotically unbiased estimator. Simulation results show that the RAWLP offers better time and frequency resolution than WLP with a fixed window support, and the regularization techniques can effectively reduce the variance of the RAWLP estimate. It is also shown in our simulation that, the time-varying harmonic and interharmonic components can be revealed satisfactorily while the noisy components are effectively suppressed by means of variable bandwidth and regularization in the RAWLP of power signals.

The paper is organized as follows. In Section II, the Lomb periodogram will be reviewed and the windowed Lomb periodogram is introduced. Section III is devoted to the proposed adaptive varying window selection method for WLP, which is followed by the proposed RAWLP in Section IV. Simulation results and comparisons are described in Section V. Finally, conclusions are drawn in Section VI.

\section{WLS-BASED WINDOWED LOMB PERIODOGRAM}

Suppose a set of discrete-time noisy samples $x_{n}$, $n=1,2, \cdots, N$, is acquired by sampling from a continuoustime signal $m(t)$,

$$
x_{n}=m\left(t_{n}\right)+\varepsilon\left(t_{n}\right)
$$

where $t_{n}$ are the sampling time instants, which may possibly be non-uniformly distributed, and $\varepsilon(t)$ is a Gaussian noise with zero mean and variance $\sigma_{\varepsilon}^{2}(t)$. The Lomb periodogram $P(\omega)$ is computed by a LS fitting of the signal $x_{n}$ by a sinusoid with frequency $\omega$. More precisely, the signal $x_{n}$ is represented by the local linear regression model:

$$
\begin{aligned}
x_{n} & =a(\omega) \cos \left(\omega t_{n}\right)+b(\omega) \sin \left(\omega t_{n}\right)+e\left(t_{n}\right) \\
& =\phi^{T}\left(t_{n}, \omega\right) \boldsymbol{\beta}(\omega)+e\left(t_{n}\right),
\end{aligned}
$$

where $x_{n}$ is the response variable, $\phi\left(t_{n}, \omega\right)=\left[\cos \left(\omega t_{n}\right), \sin \left(\omega t_{n}\right)\right]^{T}$ is the explanatory variables, and $\boldsymbol{\beta}(\omega)=[a(\omega), b(\omega)]^{T}$ is the regression coefficients. Assume $e\left(t_{n}\right)$ is a zero mean Gaussian process, the coefficient vector $\boldsymbol{\beta}(\omega)$ can be obtained by minimizing the MSE of the fitting as:

$$
\begin{aligned}
\hat{\boldsymbol{\beta}}(\omega) & =\arg \min _{\boldsymbol{\beta}} \sum_{n=1}^{N} e^{2}\left(t_{n}\right) \\
& =\arg \min _{\boldsymbol{\beta}} \sum_{n=1}^{N}\left[x_{n}-\boldsymbol{\phi}^{T}\left(t_{n}, \omega\right) \boldsymbol{\beta}(\omega)\right]^{2} \\
& =\arg \min _{\boldsymbol{\beta}}\|\boldsymbol{X}-\boldsymbol{\Phi}(\omega) \boldsymbol{\beta}(\omega)\|_{2}^{2},
\end{aligned}
$$

where $\boldsymbol{X}=\left[x_{1}, x_{2}, \cdots, x_{n}\right]^{T}$, and

$\boldsymbol{\Phi}(\omega)=\left[\begin{array}{cccc}\cos \left(\omega t_{1}\right), & \cos \left(\omega t_{2}\right), & \cdots, & \cos \left(\omega t_{N}\right) \\ \sin \left(\omega t_{1}\right), & \sin \left(\omega t_{2}\right), & \cdots, & \sin \left(\omega t_{N}\right)\end{array}\right]^{T}$. The LS

solution to above function is attained as

$$
\hat{\boldsymbol{\beta}}(\omega)=\left[\boldsymbol{\Phi}^{T}(\omega) \boldsymbol{\Phi}(\omega)\right]^{-1} \boldsymbol{\Phi}^{T}(\omega) \boldsymbol{X} .
$$

The Lomb periodogram can then be computed from the LSbased estimate of $\boldsymbol{\beta}(\omega)$ as

$$
P(\omega)=\frac{1}{N} \hat{\boldsymbol{\beta}}^{T}(\omega) \boldsymbol{\Phi}^{T}(\omega) \boldsymbol{\Phi}(\omega) \hat{\boldsymbol{\beta}}(\omega) .
$$

It can be seen that the Lomb periodogram employs all the samples in estimating the amplitude $\hat{\boldsymbol{\beta}}(\omega)$. Hence, it is unsuitable to analyze signals with time-varying amplitudes. To address this problem, we shall extend the conventional Lomb periodogram in order to reveal the time-varying spectral information. More precisely, at an given time instant $\tau$, a time window centered at $\tau$ is multiplied to the signal to extract the local spectral information. The window can be obtained by scaling a basis window function $w(\cdot)$ by a bandwidth factor of $h$, i.e., $w_{h}(t-\tau)=\frac{1}{h} w\left(\frac{1}{h}(t-\tau)\right)$. The bandwidth $h$ controls the effective length of the window and hence the number of neighboring data around $\tau$ used to estimate the periodogram. With a given bandwidth $h$ and the window support $w_{h}(t-\tau)$, the cost function of (3) and its solution is modified to

$$
\begin{aligned}
\hat{\boldsymbol{\beta}}(\tau, \omega) & =\arg \min _{\boldsymbol{\beta}} \sum_{n=1}^{N} w_{h}\left(t_{n}-\tau\right)\left[x_{n}-\boldsymbol{\phi}^{T}\left(t_{n}, \omega\right) \boldsymbol{\beta}(\tau, \omega)\right]^{2} \\
& =\arg \min _{\boldsymbol{\beta}}\|\boldsymbol{W}(\boldsymbol{X}-\boldsymbol{\Phi}(\omega) \boldsymbol{\beta}(\tau, \omega))\|_{2}^{2},
\end{aligned}
$$

where $\boldsymbol{\beta}(\tau, \omega)=[a(\tau, \omega), b(\tau, \omega)]^{T}$ is the coefficient vector and $\boldsymbol{W}=\operatorname{diag}\left\{\left[w_{h}\left(t_{1}-\tau\right), w_{h}\left(t_{2}-\tau\right), \cdots, w_{h}\left(t_{n}-\tau\right)\right]^{T}\right\}$. The resultant weighted LS (WLS) solution is:

$$
\hat{\boldsymbol{\beta}}(\tau, \omega)=\left[\boldsymbol{\Phi}^{T}(\omega) \boldsymbol{W} \boldsymbol{\Phi}(\omega)\right]^{-1} \boldsymbol{\Phi}^{T}(\omega) \boldsymbol{W} \boldsymbol{X} .
$$

By evaluating the Lomb periodogram at a set of uniformly 
distributed evaluated time instants $\tau_{m}, m=1,2, \cdots, M$, a windowed Lomb periodogram (WLP)-based TFR of the signal is obtained. A useful property of the WLP is that it is proportional to the energy concentration of the signal in the time-frequency domain, because it is calculated as the squared amplitude of a set of local sinusoids. It can also be seen that WLP is more suitable for analyzing harmonic data, since the signal is represented by a set of local sinusoids.

A major issue of the WLP method is the selection of the local adaptive bandwidth $h$. In the next section, we shall present an asymptotic analysis of the WLS estimator, and hence develop an adaptive window selection for WLP.

\section{ADAPTIVE WINDOW SELECTION IN WLP}

As mentioned before, the WLP estimation is actually a WLS estimator of the local linear regression model. In particular, the WLS estimator can be viewed as a special case of a more general local polynomial modelling (LPM) estimator for the time-varying linear regression model (TVLRM) in [20] and [21]. In fact, the WLS estimator is a LPM estimator with the polynomial order equal to zero. As the asymptotic expressions of bias and variance of the LPM estimator for the TVLRM have been derived in [20] and [21], the asymptotic bias and variance (as $N h \rightarrow \infty$ and $h \rightarrow 0$ ) of the $l$-th coefficient in the WLP estimation $\hat{\beta}_{l}(\tau, \omega)$, ( $l=1$ or $2, \hat{\beta}_{1}=\hat{a}$ and $\hat{\beta}_{2}=\hat{b}$ ), can be obtained from those in [20] and [21] as follows

$$
\begin{gathered}
\operatorname{Bias}\left(\hat{\beta}_{l}(\tau, \omega)\right)=\frac{h \widetilde{\mu}_{w}}{\mu_{w}} \beta_{l}{ }^{\prime}(\tau, \omega)+o_{P}(h), \\
\operatorname{Var}\left(\hat{\beta}_{l}(\tau, \omega)\right)=\frac{2 v_{w} \sigma^{2}(\tau)}{\operatorname{Nhf}(\tau) \mu_{w}^{2}}+o_{P}\left(\frac{1}{N h}\right),
\end{gathered}
$$

where $\mu_{w}=\int w(v) d v, \widetilde{\mu}_{w}=\int v w(v) d v, v_{w}=\int w^{2}(v) d v$, $\beta_{l}^{\prime}$ is the first derivative of $\beta_{l}, f(\tau)$ is the sampling density function at $\tau, \phi_{1}(\tau, \omega)=\cos (\omega \tau)$ and $\phi_{2}(\tau, \omega)=\sin (\omega \tau)$.

It can be seen from (8) and (9) that both the estimation bias and variance are functions of the bandwidth $h$. As $h$ increases, the squared bias will also increase while the variance will decrease. Since the mean squared error (MSE) is the sum of squared bias and variance

$$
\operatorname{MSE}\left(\hat{\beta}_{l}(\tau, \omega)\right)=\operatorname{Bias}^{2}\left(\hat{\beta}_{l}(\tau, \omega)\right)+\operatorname{Var}\left(\hat{\beta}_{l}(\tau, \omega)\right),
$$

there exists a locally optimal bandwidth $h^{+}(\tau, \omega)$, which minimizes the MSE criterion.

By setting the derivative of (10) with respect to $h$ to zero, the following optimal bandwidth at an evaluated timefrequency point $(\tau, \omega)$ is obtained:

$$
h_{l}^{+}(\tau, \omega)=\left\{\frac{v_{w} \sigma^{2}(\tau)}{4 N f(\tau)\left[\beta_{l}^{\prime}(\tau, \omega)\right]^{2} \widetilde{\mu}_{w}^{2}}\right\}^{1 / 3} .
$$

However, as some of the quantities in (11), such as $\beta_{l}{ }^{\prime}(\tau, \omega)$, are difficult to be calculated directly, the optimal bandwidth is difficult to be estimated accurately. We next introduce an empirical method to select the optimal bandwidth from a set of possible bandwidths.
In this paper, we employ the intersection of confidence intervals (ICI) method to estimate the optimal bandwidth. The ICI method is an empirical adaptive bandwidth selection method proposed by Goldenshluger and Nemirovski [12], and it has been successfully applied in various areas, such as local polynomial regression and image processing [14]-[16]. Given a set of bandwidth parameters in an ascending order:

$$
\boldsymbol{H}=\left\{h_{1}<h_{2}<\cdots<h_{J}\right\},
$$

where $J$ is the number of possible bandwidths, the ICI method determines the optimal bandwidth by comparing the confidence intervals of the estimates with different bandwidths in the set. The algorithm of ICI is omitted to save space, and more details can be found in [12]-[16].

\section{REGULARIZED AWLP}

In statistics, a regularization term is frequently used to reduce the variance in the estimation at the expense of a small extra bias. In the context of TFA of harmonic signal analysis, we are interested in lowering the variance and MSE of our WLS estimator in WLP. In the regularization, a penalty of the regression coefficient is added to the cost function to yield the following regularized WLS (RWLS) estimator ( $\tau$ and $a$ are omitted here for notational simplicity)

$$
\hat{\boldsymbol{\beta}}=\arg \min _{\boldsymbol{\beta}}\left\{\|\boldsymbol{W}(\boldsymbol{X}-\boldsymbol{\Phi} \boldsymbol{\beta})\|_{2}^{2}+\sum_{l=1}^{2} \rho_{\lambda}\left(\beta_{l}\right)\right\},
$$

where $\rho_{\lambda}(\cdot)$ is the regularization or penalty function with one or more regularization parameters $\lambda$. In practical implementation, the regularization parameters can be chosen by the generalized cross-validation criterion [17].

Some commonly-used regularization functions include the $L_{2}$ regularization $\rho_{\lambda}\left(\beta_{l}\right)=\lambda \beta_{l}^{2}$, which leads to a ridge regression [17], and the $L_{1}$ regularization $\rho_{\lambda}\left(\beta_{l}\right)=\lambda|\beta|$, which leads to a least absolute shrinkage and selection operator (lasso) [19]. In this paper, the smoothly clipped absolute deviation (SCAD) regularization function will also be investigated, because of its desirable properties: unbiasedness, sparsity, and continuity [17]. The SCAD regularization function is given as:

$$
\rho_{\lambda}\left(\beta_{l}\right)=\left\{\begin{array}{cc}
\lambda\left|\beta_{l}\right| & \text { for }\left|\beta_{l}\right| \leq \lambda, \\
-\frac{\left(\left|\beta_{l}\right|-\tilde{a} \lambda\right)^{2}}{2(\tilde{a}-1)}+\frac{(\tilde{a}+1) \lambda^{2}}{2} & \text { for } \lambda<\left|\beta_{l}\right| \leq \tilde{a} \lambda, \\
\frac{(\tilde{a}+1) \lambda^{2}}{2} & \text { for }\left|\beta_{l}\right|>\tilde{a} \lambda,
\end{array}\right.
$$

where $\tilde{a}>2$ is a tuning parameter.

Ridge, lasso and SCAD estimators all satisfy the condition of continuity, which means that $\rho_{\lambda}\left(\beta_{l}\right)$ is continuous in $\beta_{l}$. The lasso and SCAD estimators also possess the desirable property of sparsity, which means that small coefficients will be forced to zero to yield a sparse representation, while the ridge estimator does not. In the context of harmonic signal analysis, the data under study are sampled from sinusoidal signals, and hence sparse estimators are preferred as only few nonzero coefficients will be encountered. Unbiasedness implies that the modeling bias introduced by the regularization term should be zero when the coefficients are large enough. Among the three regularization techniques, only SCAD has the important property of unbiasedness. As a result, the SCAD estimator is a valuable tool for estimating 
the amplitudes of sinusoids in the harmonic analysis.

In $L_{2}$ regularization, $\rho_{\lambda}\left(\beta_{l}\right)=\lambda \beta_{l}^{2}$, and the resultant solution or the ridge estimator to the objective function in (13) is given by

$$
\hat{\boldsymbol{\beta}}=\left[\boldsymbol{\Phi}^{T} \boldsymbol{W} \boldsymbol{\Phi}+\lambda \boldsymbol{I}\right]^{-1} \boldsymbol{\Phi}^{T} \boldsymbol{W} \boldsymbol{X} .
$$

For lasso and SCAD, the objective functions are nondifferentiable and it is difficult to obtain their solutions in analytic form. An iteratively re-weighted least-squares (IRLS) algorithm for solving this non-differentiable function was proposed in [19] and [17]. Due to page limitation, the details of the IRLS algorithm are omitted here, and they can be found in [19] and [17].

We now summarize the proposed regularized AWLP (RAWLP) method as follows.

Step 1. At each evaluated time $\tau$ and frequency $a$, the coefficient $\hat{\boldsymbol{\beta}}\left(\tau, \omega, h_{j}\right)$ is calculated by the WLS estimator (7) with each bandwidth $h_{j}$ in the set $\boldsymbol{H}$ of (12).

Step 2. A optimal bandwidth $h^{+}(\tau, \omega)$ is estimated using the ICI method based on the estimates $\hat{\boldsymbol{\beta}}\left(\tau, \omega, h_{j}\right)$, $j=1, \cdots, J$.

Step 3. The final estimate of the time-varying coefficient $\hat{\boldsymbol{\beta}}\left(\tau, \omega, \tilde{h}^{+}(\tau, \omega)\right)$ is obtained using the RWLS estimation (13) with bandwidth $\widetilde{h}^{+}(\tau, \omega)$.

We now discuss several practical issues of the RAWLP method. Firstly, following the recommendation of literature [20] and [21], the Epanechnikov window is employed, because of its good theoretical performance:

$$
w(u)=\left\{\begin{array}{cc}
\frac{3}{4}\left(1-|u|^{2}\right) & |u|<1, \\
0 & |u| \geq 1 .
\end{array}\right.
$$

Consequently, for an Epanechnikov window with bandwidth $h$, only the data samples included in interval $(\tau-h, \tau+h)$ are used and thus the effective length of the window is $2 h$.

The next problem is how to select the bandwidth set $\boldsymbol{H}$ used in the empirical ICI bandwidth selection method. The minimum bandwidth $h_{1}$ should be selected to make the WLS estimators solvable. More precisely, the number of data samples included in the interval $\left(\tau-h_{1}, \tau+h_{1}\right), N_{w}$, should be equal to or larger than 2 (the number of coefficients). On the other hand, the largest element in the bandwidth set, $h_{J}$, should be large enough to include all data points. However, too long a window will result in high computational complexity. In practical implementation, $h_{1}$ and $h_{J}$ can be determined based on known or interested time-frequency properties of the signals. As for other bandwidths in the set $\boldsymbol{H}$, more bandwidths may lead to more refined results, but will also increase the complexity. To achieve a tradeoff between performance and complexity, we generally select 3-5 bandwidth parameters between $h_{1}$ and $h_{J}$. From simulation results, it was found that the proposed bandwidth setting gave satisfactory results.

\section{SimUlation RESUlts}

\section{A. Simulated Chirp Signals}

We first compare the various TFA methods for a simulated uniform data sampled from a chirp signal

$$
m(t)=A(t) \sin (\Omega(t)+\varphi),
$$

where $A(t)=1$ is the amplitude, $\varphi=\pi / 3$ is the phase, and $\Omega(t)=2 \pi \int_{0} f(v) d v$ is the angular frequency calculated from an instantaneous frequency $f(t)$. Here, $f(t)$ is given as

$$
f(t)=25+7 \operatorname{asinh}(8(t-1))
$$

where $\operatorname{asinh}(\cdot)$ is the inverse hyperbolic sine function. The data duration is 2 seconds and the sampling frequency is $100 \mathrm{~Hz}$, resulting in 200 data samples. An additive Gaussian white noise with a signal-to-noise ratio (SNR) of $10 \mathrm{~dB}$ is added. One realization of the uniformly sampled data and the instantaneous frequency are shown in Fig. 1. We can see that this signal contains both slowly-varying harmonics and fastvarying harmonics, and so it can serve as a good example for evaluating the performance of the various Lomb periodogram-based TFA methods.

The WLS-based AWLP and the RWLS (including ridge, lasso, and SCAD)-based RAWLP with varying bandwidth is compared to WLP with a constant bandwidth. For comparison, the WT with Morlet wavelet is also tested. In the WLP, the bandwidth set used in the ICI method is chosen as $\boldsymbol{H}=\{0.1,0.15,0.2,0.25\}$ seconds , which corresponds to effective window lengths $\{0.2,0.3,0.4,0.5\}$ seconds. The evaluated time instants are set as the sampling time instants, and the evaluated frequency instants are from $0.5 \mathrm{~Hz}$ to $50 \mathrm{~Hz}$ with a frequency step of $0.5 \mathrm{~Hz}$.

One representative simulation is presented in Fig. 1, where the Lomb periodogram based TFRs are shown in the same scale (the maximum possible value of these TFRs is calculated as $\left.\max (P(\tau, \omega))=\max \left(|A(t)|^{2}\right)=1\right)$. It can be seen from Fig. 1 that,

1) If a small bandwidth is used, the WLP can identify the fast-varying harmonic satisfactorily, which implies good time resolution. But the spectral density of slowlyvarying harmonic is dispersed along the frequency domain, leading to rather poor frequency resolution. On the other hand, if a large bandwidth is used, the frequency resolution for slowly-varying harmonic is considerably increased. However, the fast-varying harmonic is blurred seriously due to the decreased time resolution.

2) The ICI method can adaptively select the bandwidth based on the time-frequency localization of the signal. The bandwidths for fast-varying harmonics are small, while large bandwidths are assigned to slowly-varying harmonics. For time-frequency areas where there are no meaningful frequency components, the bandwidths are given the largest value to restrain the effect of noise. With the varying bandwidth, the AWLP achieves a better TFR than WLP with a constant bandwidth. The AWLP also outperforms the WT, which has bad time resolution for low-frequency components and bad frequency resolution for high-frequency components. 

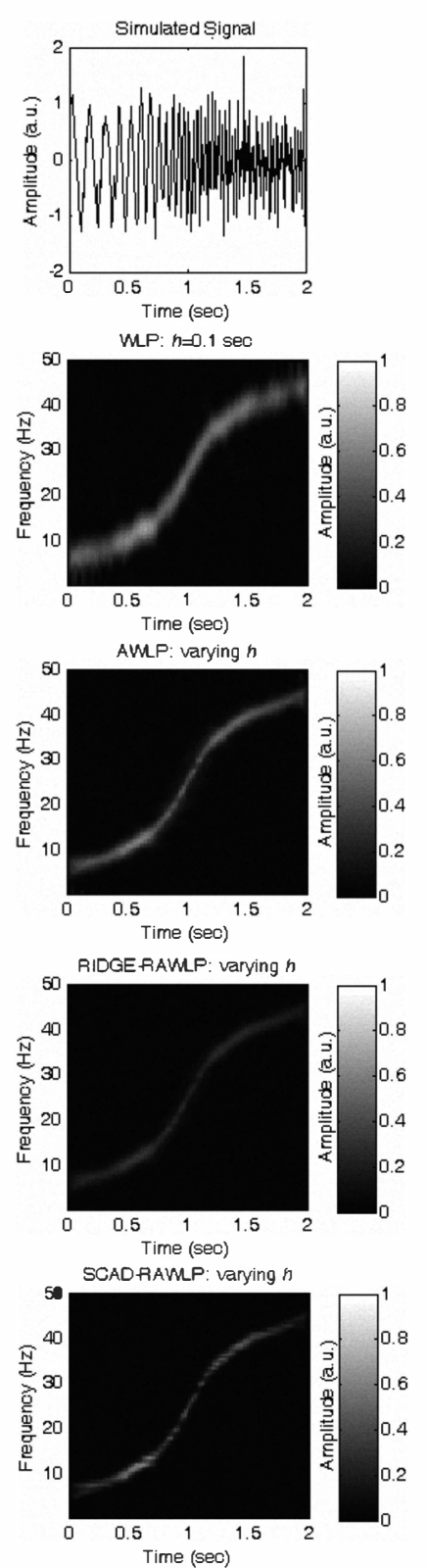

Fig. 1. An example of uniformly sampled signal and its time-frequency representations using different TFA methods.

3) Regularization techniques can further lower the variance of the estimator and the three RWLS-based RAWLPs have better time-frequency resolution than the WLSbased AWLP. However, it can also be seen that the ridge and lasso estimators produce considerable bias to the large spectral coefficients, and that the resultant ridge and lasso-based RAWLP have lower peak values than the WLS-based AWLP. The SCAD-based RAWLP has peak values comparable to the WLS-based AWLP.

We next select a set of time-frequency points, which have different but representative time-frequency features for a more quantitative comparison between different TFA methods. These time-frequency points are labeled as p1 to p5 in the upper left panel of Fig. 2. The values of the periodogram at these five time-frequency points are collected, and the amplitudes of sinusoids at these points are estimated
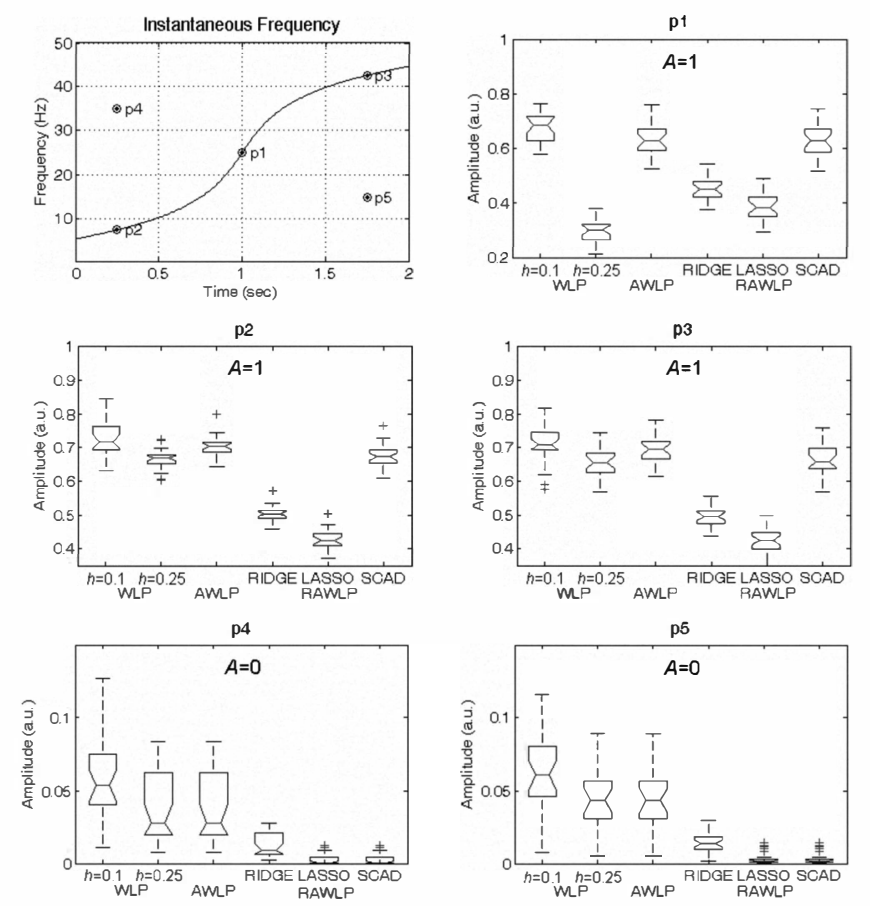

Fig. 2. Comparison of amplitude estimates using different Lombperiodogram-based TFA methods. The box has lines at the lower quartile, median (red), and upper quartile values. Whiskers extend from each end of the box to the most extreme data values.

as $\hat{A}(\tau, \omega)=\sqrt{P(\tau, \omega)}$. The true amplitudes at timefrequency points from $\mathrm{p} 1$ to $\mathrm{p} 5$ are respectively: $A=1,1,1,0$, 0 . The results of 100 Monte-Carlo simulations are presented in box plots of Fig. 2 with the following observations.

1) By comparing the WLP results at p1, p2 and p3 using $h=0.1$ and $h=0.25$, we can see that a small bandwidth results in a large variance and a small bias, while a large bandwidth leads to a large bias and a small variance. The good bandwidth selected by the ICI method can achieve a compromise between bias and variance.

2) Compared with the WLS-based AWLP, the ridge, lasso and SCAD-based RAWLPs all exhibit extra bias for large amplitudes (at p1, p2 and p3). Among the three regularized estimators, lasso has the largest variance while SCAD has the smallest bias because SCAD is an asymptotically unbiased estimator.

3) As for the variance, both ridge and lasso estimators have a lower variance than the WLS-based AWLP, and the variance of the ridge estimator is lower than that of lasso. However, SCAD estimator shows a slightly increased variance, which may be due to the difficulty in choosing the right regularization parameters.

4) For small coefficients caused by noise components ( $\mathrm{p} 4$ and $\mathrm{p} 5$ ), the lasso and SCAD estimators can shrink them to zero, which is desirable. Ridge estimator can also reduce the estimation variance to some extent.

\section{B. Interharmonic Estiamtion in Power Systems}

Next, we apply the proposed RAWLP method to the estimation of the interharmonics in a simulated power supply system. A 200-ms data sequence with a sampling rate of 200 $\mathrm{kHz}$ and a SNR of $15 \mathrm{~dB}$ was generated to simulate the current signals from a fluorescent lamp with a high-frequency 
ballast. The waveform and its interharmonic frequency are respectively shown in Fig. 3. In the WLP, the bandwidth set is chosen as $\boldsymbol{H}=\{0.05,0.1,0.2,0.5\} \mathrm{ms}$. The evaluated time instants are set as the sampling time instants, and the evaluated frequency instants are from $1 \mathrm{kHz}$ to $10 \mathrm{KHz}$ with a frequency step $1 \mathrm{kHz}$. Similarly to our observation in the previous simulation, Fig. 3 shows that, a small window lead to poor frequency resolution while a large window results in poor time resolution. The AWLP method is able to achieve good tradeoff between time and frequency resolution. Moreover, the SCAD-based RAWLP can identify the interharmonic frequency more accurately due to its important properties of unbiasedness and sparsity.

\section{CONCLUSION}

A new regularized adaptive windowed Lomb periodogram (RAWLP) method for TFA of non-stationary harmonic signals is presented. Firstly, the conventional Lomb periodogram is extended to an adaptive windowed Lomb periodogram (AWLP) by means of a sliding-window with variable bandwidths. Secondly, the window length or bandwidth is adaptively selected by the ICI method to adapt to local time-frequency characteristics of the signals. Thirdly, the regularization techniques are incorporated to further improve the performance of the AWLP by reducing the variance of the estimator. Simulation results show that the propose RWALP method is an effective TFA method for analyzing harmonic signals and it can find applications in power signal processing. The proposed method can also be extended to the analysis of multi-component signals [22] and the results will be reported elsewhere.

\section{REFERENCES}

[1] B. Boashash, Time Frequency Signal Analysis: Methods and Applications. Melbourne: Longman Cheshire, 1992.

[2] M. Akay, Time-Frequency and Wavelets in Biomedical Signal Processing. Piscataway, NJ: IEEE Press, 1997.

[3] T. F. Quatieri, Discrete-Time Speech Signal Processing: Principles and Practice. Upper Saddle River, NJ: Prentice Hall, 2002.

[4] Y. H. Gu and M. H. J. Bollen, "Time-frequency and time-scale domain analysis of voltage disturbances," IEEE Trans. Power Deliv., vol. 15, no. 4, pp.1279-1284, 2000.

[5] P. K. Dash and M. V. Chilukuri, "Hybrid S-transform and Kalman filtering approach for detection and measurement of short duration disturbances in power networks," IEEE Trans. Instrum. Meas., vol. 53, no. 2, pp. 746-753, April 2004.

[6] I. Y. H. Gu and M. H. J. Bollen, "Estimating interharmonics by using sliding window ESPRIT," IEEE Trans. Power Deliv., vol. 23 , no. 1, pp 13-23, 2008.

[7] M. H. J. Bollen, I. Y. H. Gu, S. Santoso, M. F. McGranaghan, P. A. Crossley, M. V. Ribeiro, and P. F. Ribeiro, "Bridging the gap between signal and power," IEEE Signal Process. Mag., vol. 26, no. 4, pp. 12$31,2009$.

[8] N. R. Lomb, "Least-squares frequency analysis of unequally spaced data," Astrophys. Space Sci., vol. 39, pp. 447-462, 1976.

[9] J. D. Scargle, "Studies in astronomical time series analysis II: Statistical aspects of spectral analysis of unevenly sampled data," Astrophys. J., vol. 263, pp. 835-853, 1982.

[10] P. Laguna, G. B. Moody, and R. G. Mark, "Power spectral density of unevenly sampled data by least-square analysis: Performance and application to heart rate signals," IEEE Trans. BioMed. Engg., vol. 45, no. 6, pp. 698-715, June 1998.

[11] E. F. Glynn, J. Chen, A. R. Mushegian, "Detecting periodic patterns in unevenly spaced gene expression time series using Lomb-Scargle periodograms," Bioinformatics vol. 22, no. 3, pp. 310-316, 2006.

[12] Z. G. Zhang and S. C. Chan, "Robust adaptive Lomb periodogram for time-frequency analysis of signals with sinusoidal and transient
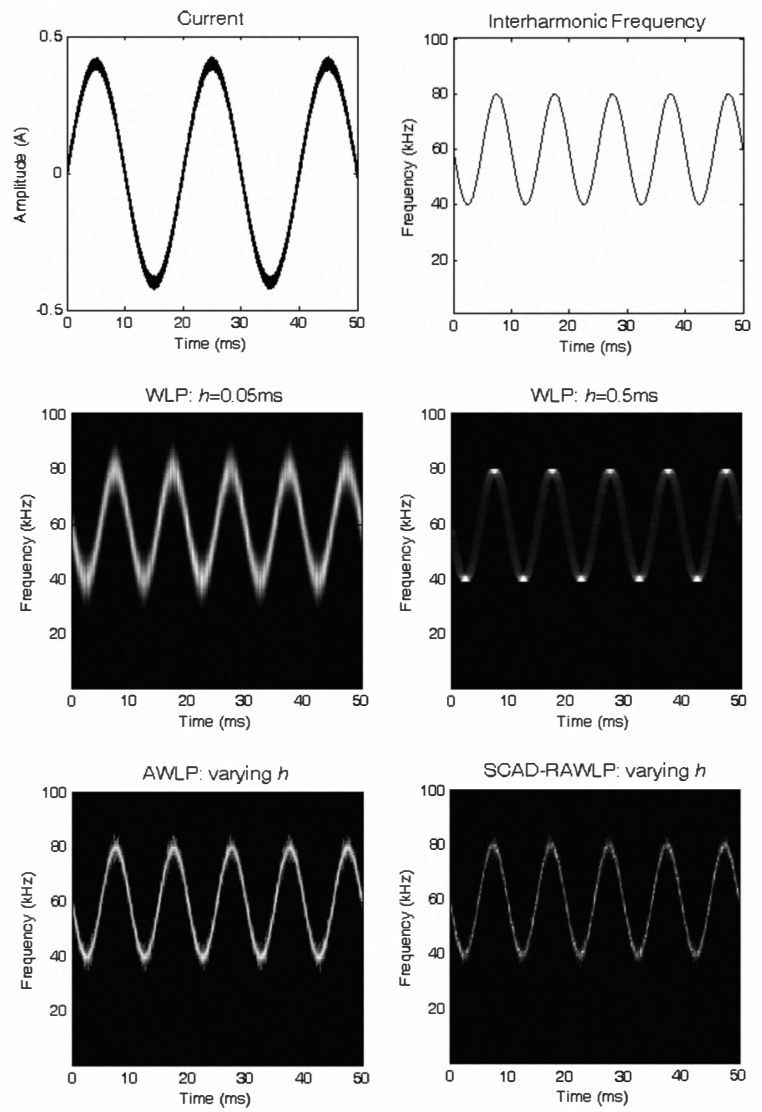

Fig. 3. A simulated power supply current signal from a fluorescent lamp and its time-frequency representations using different TFA methods.

components," in Proc. IEEE International Conference on Acoustics, Speech, and Signal Processing (ICASSP 2005), Philadelphia, PA, USA, 18-23 March, 2005, vol. 4, pp. 493-496.

[13] A. Goldenshluger and A. Nemirovski, "On spatial adaptive estimation of nonparametric regression," Math. Methods Statist., vol. 6, no. 2, pp. 135-170, 1997.

[14] V. Katkovnik, K. Egiazarian, and J. Astola, Local Approximation Techniques in Signal and Image Processing. Bellingham: SPIE Press, Sept. 2006.

[15] Z. G. Zhang, S. C. Chan, K. L. Ho, and K. C. Ho, "On bandwidth selection in local polynomial regression analysis and its application to multi-resolution analysis of non-uniform data," J. Signal Process. Syst., vol. 52, no. 3, pp. 263-280, Sept. 2008.

[16] Z. G. Zhang, S. C. Chan, and Z. Y. Zhu, "A new two-stage method for restoration of images corrupted by Gaussian and impulse noises using local polynomial regression and edge preserving regularization," in Proc. IEEE International Symposium on Circuits and Systems (ISCAS 2009), Taipei, 24-27 May, 2009, pp. 948-951.

[17] J. Fan and R. Li, "Variable selection via nonconcave penalized likelihood and its oracle properties,". J. Am. Stat. Assoc., vol. 96, no. 456 , pp. 1348-1360, Dec. 2001.

[18] A. N. Tikhonov and V. Y. Arsenin, Solutions of Ill-Posed Problems. Washington, DC: Winston and Sons, 1977.

[19] R. Tibshirani, "Regression shrinkage and selection via the lasso," $J$. Royal. Statist. Soc B., vol. 58, no. 1, pp. 267-288, 1996.

[20] S. C. Chan and Z. G. Zhang, "Local polynomial modeling and bandwidth selection for time-varying linear models," in Proc. International Conferences on Information, Communications and Signal Processing (ICICS 2009), Macau, China, 7-10 Dec, 2009.

[21] Z. G. Zhang, S. C. Chan, and Y. S. Hung, "Local polynomial modelling of time-varying autoregressive processes and its application to the analysis of event-related electroencephalogram," in Proc. IEEE International Symposium on Circuits and Systems (ISCAS2010), Paris, France, 30 May-2 June, 2010.

[22] S. C. Chan, "Bayesian spectral estiamtion," Internal Report. Department of Electrical and Electronic Engineering, The University of Hong Kong, 2009. 